University of New Hampshire

University of New Hampshire Scholars' Repository

$9-2006$

\title{
Self-Positioning Smart Buoys, The 'Un-Buoy' Solution: Logistic Considerations Using Autonomous Surface Craft Technology and Improved Communications Infrastructure
}

\author{
Joseph A. Curcio \\ Massachusetts Institute of Technology \\ Philip A. McGillivary \\ US Coast Guard \\ Kevin Fall \\ Woods Hole Oceanographic Institution \\ Andrew Maffei \\ Woods Hole Oceanographic Institution \\ Kurt Schwehr \\ University of New Hampshire, Durham \\ Follow this and additional works at: https://scholars.unh.edu/ccom \\ Part of the Computer Sciences Commons, and the Oceanography and Atmospheric Sciences and \\ See next page for additional authors \\ Reteorology Commons
}

\section{Recommended Citation}

Curcio, Joseph A.; McGillivary, Philip A.; Fall, Kevin; Maffei, Andrew; Schwehr, Kurt; Twiggs, Bob; Kitts, Chris; and Ballou, Phil, "Self-Positioning Smart Buoys, The 'Un-Buoy' Solution: Logistic Considerations Using Autonomous Surface Craft Technology and Improved Communications Infrastructure" (2006). IEEE Oceans. 366.

https://scholars.unh.edu/ccom/366

This Conference Proceeding is brought to you for free and open access by the Center for Coastal and Ocean Mapping at University of New Hampshire Scholars' Repository. It has been accepted for inclusion in Center for Coastal and Ocean Mapping by an authorized administrator of University of New Hampshire Scholars' Repository. For more information, please contact Scholarly.Communication@unh.edu. 


\section{Authors}

Joseph A. Curcio, Philip A. McGillivary, Kevin Fall, Andrew Maffei, Kurt Schwehr, Bob Twiggs, Chris Kitts, and Phil Ballou 


\title{
Self-Positioning Smart Buoys, The "Un-Buoy" Solution: Logistic Considerations using Autonomous Surface Craft Technology and Improved Communications Infrastructure
}

\author{
Joseph A. Curcio, Massachusetts Institute of Technology, Cambridge, MA \\ Philip A. McGillivary, USCG PACAREA Science Liaison, Alameda, CA \\ Kevin Fall, Intel Research Berkeley, Berkeley, CA \\ Andy Maffei, Woods Hole Oceanographic Institution, Woods Hole, MA \\ Kurt Schwehr, Center for Coastal and Ocean Mapping, Univ. of New Hampshire, Durham, NH \\ Bob Twiggs, Department of Aeronautics and Astronautics, Stanford University, Stanford, CA \\ Chris Kitts, Santa Clara University, Santa Clara, CA \\ Phil Ballou, Ocean Systems, Inc., Alameda, CA
}

\begin{abstract}
Moored buoys have long served national interests, but incur high development, construction, installation, and maintenance costs. Buoys which drift off-location can pose hazards to mariners, and in coastal waters may cause environmental damage. Moreover, retrieval, repair and replacement of drifting buoys may be delayed when data would be most useful. Such gaps in coastal buoy data can pose a threat to national security by reducing maritime domain awareness. The concept of self-positioning buoys has been advanced to reduce installation cost by eliminating mooring hardware. We here describe technology for operation of reduced cost self-positioning buoys which can be used in coastal or oceanic waters. The ASC SCOUT model is based on a selfpropelled, GPS-positioned, autonomous surface craft that can be pre-programmed, autonomous, or directed in real time. Each vessel can communicate wirelessly with deployment vessels and other similar buoys directly or via satellite. Engineering options for short or longer term power requirements are considered, in addition to future options for improved energy delivery systems. Methods of reducing buoy drift and positionmaintaining energy requirements for self-locating buoys are also discussed, based on the potential of incorporating traditional maritime solutions to these problems. We here include discussion of the advanced Delay Tolerant Networking (DTN) communications draft protocol which offers improved wireless communication capabilities underwater, to adjacent vessels, and to satellites. DTN is particularly adapted for noisy or loss-prone environments, thus it improves reliability. In addition to existing buoy communication via commercial satellites, a growing network of small satellites known as PICOSATs can be readily adapted to provide low-cost communications nodes for buoys. Coordination with planned vessel Automated Identification Systems (AIS) and International Maritime Organization standards for buoy and vessel notification systems are reviewed and the legal framework for deployment of autonomous surface vessels is considered.
\end{abstract}

\section{INTRODUCTION}

A range of applications exist for devices which can augment functions provided by traditional moored oceanographic buoys.
Recently developed station keeping autonomous buoy platforms may be particularly useful for situations where rapid buoy platform deployment is required [1],[2]. Other justifications for self-positioning buoys include increased mobility of these platforms, dynamic network capability (i.e. the ability to interact with each other in response to ambient conditions or directives), and reducing deployment and maintenance costs.

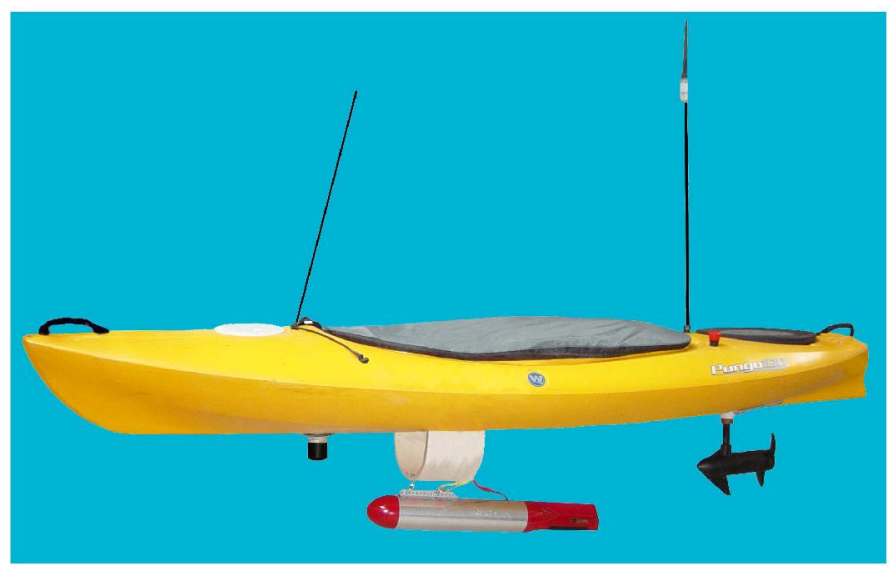

Figure 1. ASC SCOUT Vehicle outfitted with acoustic modem, side scan sonar, GPS, RF communications hardware, wireless Ethernet.

The SCOUT Autonomous Surface Craft (ASC) "Fig. 1" recently developed at MIT has successfully conducted field demonstrations of the hardware necessary for controlled propulsion and navigation essential for a station-keeping buoy [3],[4]. In addition, the open source MOOS software suite employed on SCOUT has been used with both autonomous underwater vehicles and autonomous surface craft in a wide variety of applications, including station keeping tasks [5]. The addition of oceanographic, meteorological or other 
sensors would allow SCOUT to remain un-tethered while performing the functions of a moored buoy. Long range energy efficient radio communications hardware would allow the SCOUT to transmit data to a land, ship or satellite-based data collection nodes. The inherent mobility of such a platform would allow for dynamic re-positioning.

Among the advantages gained by deploying an autonomous station-keeping buoy "Fig. 2," is the ability to track mobile or episodic events. Also, multiple buoys can be self-directed to form a network providing regional coverage or to establish and maintain perimeters. Due to the network infrastructure, individual robots empowered only to detect and/or monitor a relatively narrow physical space can thus be more effective as part of a larger awareness domain. Use of multiple selfpositioning vessels thus leverages individual assets in a collaborative fashion to produce surveillance results not achieved as effectively by even a large population of stationary and solitary sentinels.

Deployment of multiple un-tethered self-positioning vessels in conjunction with traditional moored buoys may offer another potentially useful operational scenario. Moored buoys offer certain advantages over their un-tethered counterparts, including: the ability to ride out rough weather with minimal expended energy, larger power storage capacity, reliable communications to and from land-based stations, and the elimination of sophisticated navigation and control software. These advantages weigh in against drawbacks including: fabrication and maintenance costs, deployment costs, limited effective sensor range, and the potential for entanglement and/or loss due to weather and collisions. However, let us consider a hybrid approach whereby we deploy a single moored buoy which can dock with multiple low-cost highly mobile vehicles with position-keeping capabilities. The docking buoy could provide adequate power for recharging the mobile assets, act as a relay station with increased power for increased wireless range, and permit the potentially more vulnerable mobile assets to dock and maintain position during inclement weather without expending significant energy.

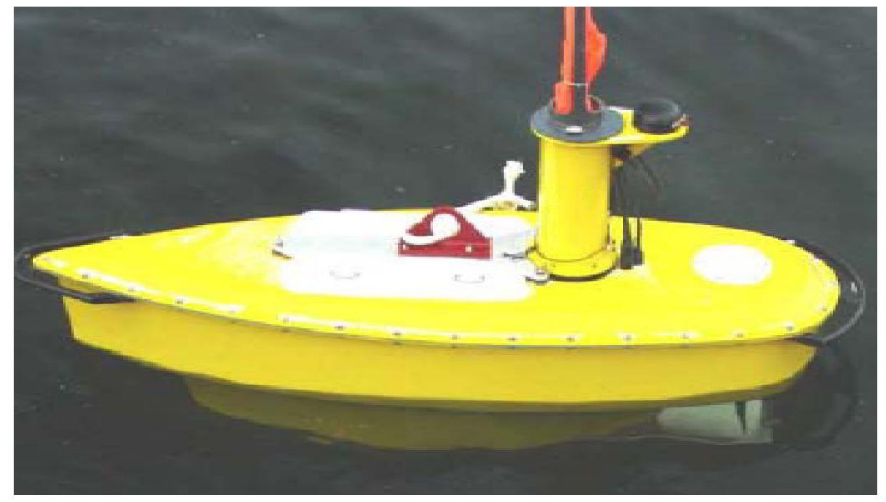

Figure 2. NUWC Station keeping buoy. Photo courtesy DARPA.
The hybrid moored buoy/mobile platform approach might prove itself most valuable in areas where oceanographic, biological or manmade events occur with some unpredictable frequency over a variable spatial region. Such events include coastal freshwater runoff and upwelling events, both of which may disperse unpredictably. In such areas a moored docking buoy could be outfitted with several autonomous mobile buoys which perform routine patrols to detect initiation of plumes associated with events. Upon detection, additional mobile buoys could be launched to assist in mapping and characterization of the phenomenon. This arrangement is similar to the concept of a small scale ocean observatory outfitted with multiple autonomous surface craft as depicted in "Fig. 3".

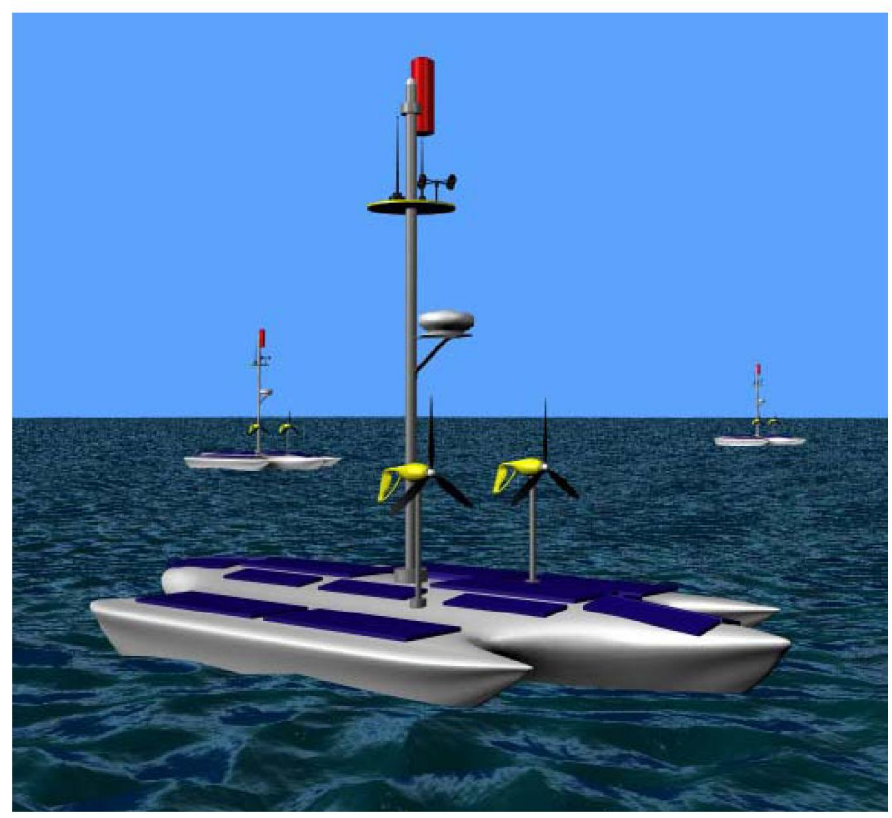

Figure 3. ASC Sea Rover concept vehicle. Courtesy NASA Ames Research Center, Jesse Haitt, UCSC.

\section{ENERGy CONSIDERATIONS FOR SELF-POSITIONING VESSELS.}

The ASC SCOUT self-propelled autonomous surface vehicle developed at MIT [3],[4] employs an electric trolling motor for propulsion, and sealed lead-acid batteries for power storage. Autonomous control is provided through a custombuilt single board computer with communications to shore handled through an RF Modem or standard 802.11 wireless Ethernet architecture. Standard R/C control allows for local remote maneuvering and operation. To date, ten SCOUT vehicles have been operated in lakes and oceans for applications including mine detection, collaborative behavior studies, collision avoidance testing, acoustic communications, and AUV positioning. Design iterations to the original vehicle have produced a more robust electrical infrastructure, longer range radio communication capability, higher operating speeds, and more extensive payload capability. 
The current ASC SCOUT vehicle stores power in a bank of AGM (Absorbed Glass Mat) batteries selected for low cost and availability. This bank is rated at approximately $100 \mathrm{Amp}-$ hours, which satisfied design intentions of operating at 3 knots for 8 hours duration. In the present configuration, the ASC SCOUT typically consumes roughly 400 Watts of power while underway at 3 knots, with the onboard computer processing data and continuously transmitting via wireless Ethernet. The power budget for the SCOUT vehicle breaks down roughly as: thruster at full speed $=300 \mathrm{~W}$; main vehicle computer $=60 \mathrm{~W}$; communications and navigation $=25 \mathrm{~W}$; miscellaneous $=15 \mathrm{~W}$. By reducing the thrust duty cycle and regulating other on board systems, numerous field deployments have demonstrated that these vehicles can operate for up to eight hours with thrusters operating at least $50 \%$ of the time. For purposes of discussion, we will consider station keeping with $10 \%$ propulsive duty cycle, approximately 30 Watts per hour over a 24 -hour period. In order to achieve higher speed for longer duration, several energy storage system modifications are under consideration for the ASC SCOUT, including use of: solar photovoltaic panels; diesel/electric propulsion systems; and, wave and/or wind energy harvesting technologies.

Typical photovoltaic solar energy collectors are now capable of harnessing and providing on order of $10 \%$ of the theoretically available energy from the sun. Published PV efficiency values vary based on factors including; location on the earth, collector technology, orientation, surface temperature, and so on. In general, while the sun irradiates the earth with roughly 1000 Watts/meter ${ }^{2}$, one typical PV solar panel suitable for maritime applications will produce on order of 50 to $60 \mathrm{Watts} / \mathrm{meter}^{2}$, (roughly $5.5 \%$ efficiency) (USF 32PV Cell from King Solar LLC, West Linn, Oregon, USA). Whereas the ASC SCOUT vehicle has an available deck surface of approximately 0.78 meter $^{2}$, we should expect to be able to harness approximately 42 Watts in full, direct sunlight. Operating in the open ocean at mid latitudes in summer, one might expect to collect energy for 8 to 10 hours at perhaps 40 Watts, for a day total of around 320 to 400 Watt hours, far too low to provide any significant contribution to our total daily power needs.

Because the ASC SCOUT vehicle operates at the surface, it can employ internal or external combustion engines, such as a small diesel/electric power plant. As noted above, the ASC SCOUT vehicle can be effectively operated using 100 Watts per hour. The existing battery pack will store approximately 1200 Watt hours of energy, or half of daily requirements under these operational parameters. A small $0.5 \mathrm{HP}$ diesel engine operating at $80 \%$ efficiency will output roughly 300 Watts of power. Operating this generator for 8 hours per day would satisfy vessel daily demand for 2400 Watt Hours of energy. Using a typical SFC (Specific Fuel Consumption) of 0.4 $\mathrm{lb} /(\mathrm{hp} \mathrm{hr}$ ) for diesel fuel, operating for 8 hours, would require approximately $1.6 \mathrm{lbs}$ of fuel: consumption of approximately 0.23 US Gallons per day. (Diesel fuel weighs approximately $7.1 \mathrm{lbs} . / \mathrm{US}$ gallon). Equipped with a $10 \mathrm{US}$ Gallon fuel reservoir, we will theoretically be able to operate SCOUT for approximately 44 days. The fuel will burden the vehicle with an additional payload of approximately $71 \mathrm{lbs}$. and added infrastructure weight of up to $50 \mathrm{lbs}$., within SCOUT's safe working payload capacity of $175 \mathrm{lbs}$. Conservatively, this should meet the objective for a 30 day deployment dedicated primarily to a station keeping mission.

\section{ADVANCED COMMUNICATION AND IDENTIFICATION PROTOCOLS}

Safe operation [6],[7] and reliable communication are integral to the success of station keeping buoys operating autonomously in open water [8]. An improved wireless methodology currently under development by engineers at Intel Research in Berkeley, CA holds promise as a tool to improve wireless communications in the marine environment. In coastal deployments ASCs can communicate directly with ships or shore stations, while further offshore communications via satellite are possible. Transmission hardware and power requirements have dropped in recent years allowing a relatively small platform with limited energy resources to include satellite transmission capability. Communications using recently introduced AIS vehicle identification systems further improves the safety of un-tethered autonomous buoys by providing positional information to other vessels.

\section{A. Delay Tolerant Networking}

Wireless line of sight transmission is substantially degraded when operating over water. Incomplete data transmissions correlate directly with increased energy usage, as well as the possibility of data loss. In an effort to create a more common and reusable system for handling intermittent networks, a Delay Tolerant Networking (DTN) Architecture is being developed within the Internet Engineering Task Force's Delay Tolerant Networking Research Group [9]. Members have developed a DTN reference implementation - software capable of handling network message routing over diverse networks (including Internet and non-Internet like networks) where disconnection is a comparatively normal occurrence. DTN operates as a network overlay, meaning it can provide a data forwarding service over heterogeneous networks. It uses persistent storage available in the network (disk, flash, etc.) to store data while network links are not available. The opensource DTN reference implementation includes a set of existing applications, a library for writing new applications, and a forwarding agent which handles message routing and provides persistent storage. The key advantages of using DTN are increased communications reliability and minimal energy consumption. 


\section{B. Satellite communication interface}

Over the past decade programs to extend educational outreach materials for satellite technology have proliferated. So-called nano-satellite programs have evolved to pico-satellite programs [10],[11]. Construction and launching of small satellites has become an active part of graduate courses in more than a dozen countries [12]; current databases at http://centaur.sstl.co.uk/SSHP/micro/index.html and http://centaur.sstl.co.uk/SSHP/nano/index.html. Whereas relatively inexpensive commercially available kits for constructing small communication satellites are now available (see http://www.pumpkininc.com), and mechanisms are in place to coordinate launch logistics as ancillary payloads on commercial satellite launch vehicles, their use is likely to increase. In the near future, deployment of these satellites with improved batteries will lengthen the lifetime of these satellites from the current endurance of one to several years to decades.

Once launched these small satellites provide free satellite communications. In the current store-and-forward configuration, the satellites transmit only when over a ground station and the communication rate of these satellites is limited by their onboard data storage. Soon, however, improved communication methods will allow communication of data between these small satellites, facilitating so-called 'bent pipe' data transmission whereby data is communicated from one satellite to another which can transmit to a ground receiving station. In the bent-pipe configuration bandwidth is limited not by storage on the satellite, but rather by the communication channel capacity, and, importantly there is a reduction in communications latency, which is important for operational control of vessels in near-real time. As DTNbased communications become increasingly used on these small satellites, and data communications between satellites becomes more commonplace, they will provide useful links for data communications for autonomous surface craft.

\section{Automatic Vessel Identification System (AIS)}

Increased need for rapid, complete port and harbor security has led to a plan for enhanced development of the so-called maritime vessel Automatic Identification System (AIS). The system under development uses a local VHF network to provide shore stations the ability to exchange low bandwidth messages with vessels approaching a port to confirm information on position and security-related data.

AIS has recently been extended to include communications not only for manned vessels, but for moored buoys and selfpositioning autonomous vessels as well. This allows these vessels to operate in full accordance with established legal requirements for maritime vessels. AIS communication ranges can be extended by piggy-backing communications via other ships capable of DTN communications. Once an AIS packet has been transferred by DTN to a ship, the system can decode the message and inject the appropriate serial messages into ships' Electronic Chart Display and Information System (ECDIS) such that the display is indistinguishable from messages received over the standard ship AIS network. The ability of an ASC SCOUT outfitted with an AIS transceiver to comply with AIS communications protocols allows the ASC to not only transmit its' location to local vessels so that these vessels can avoid collisions with it, but likewise the ASC SCOUT can receive local vessel traffic messages, and, if warranted, automatically take evasive action to protect itself from being run over by other vessels.

One of the authors (Schwehr) is proposing an open source XML-based definition language that allows vendors to rapidly build and test software compliant with new message capabilities that will extend AIS to carry additional data. With the advent of capabilities such as AIS over DTN, a network of ASCs would be able to transmit not just standard AIS data, but also all sensor data to any vessel or station requiring data.

\section{CONCLUSION}

The ASC SCOUT continues to undergo development and improvement. The MIT team has added an autonomous winch package into four of the ASC SCOUT vehicles that will be used to deploy a CTD (conductivity, temperature and depth) sensor package (SeaBird SBE-49) as well as acoustic modems mounted in custom built tow fish housings. Plans are underway to include wave and wind measurement sensors and extended range communications hardware. Constant software evolution is improving overall system robustness and reliability. In order to further improve the basic ASC SCOUT for use as a station keeping buoy, alternate hull forms are being evaluated, including SWATH (Small Water-plane Area Twin Hull) options, shown in "Fig. 4". Lastly, autonomous docking and alternative power systems are being explored as components for future development.

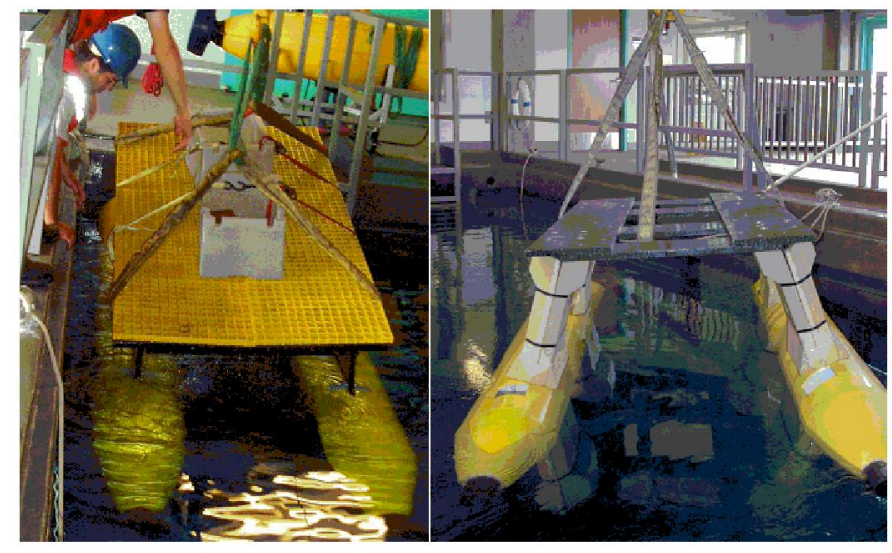

Figure 4.The MBARI Santa Clara University SWATH (Small Waterplane Area Twin Hull) vehicle 


\section{ACKNOWLEDGMENT}

The authors would like to acknowledge support from the NOAA Office of Coastal Survey (Schwehr), and helpful discussion with the members of the Delay Tolerant Networking Research Group (DTNRG), and the National AIS Network Group at the US Coast Guard Research and Development Center (Groton, Conn.). Additional useful discussions have been ongoing with Jim Bellingham and Bill Kirkwood of the Monterey Bay Aquarium Research Institute (MBARI), Tom Curtin of the Office of Naval Research (ONR) and Marc Stewart of The Applied Physics Laboratory, University of Washington.

\section{REFERENCES}

[1] Manley, J., Marsh, A., Cornforth, W., and Wiseman. Evolution of the autonomous surface craft Autocat. IEEE Oceans, 2000.

[2] Curtin, T., Crimmins, D., Curcio, J., Benjamin, M.,Roper, C., Autonomous Underwater Vehicles: Trends and Transformations" The Marine Technology Society Journal, Volume 39, Number 3, Fall 2005.

[3] Curcio, J., Leonard, J., Patrikalakis, A. 2005. "SCOUT - A Low Cost Autonomous Surface Craft for Research in Cooperative Autonomy", in IEEE Oceans, 2005.
[4] Curcio, J., Leonard, J., Vaganay., Patrikalakis, A., Bahr, A., Battle, D., Schmidt, H., Grund, M. 2005. "Experiments in Moving Baseline Navigation using Autonomous Surface Craft", in IEEE Oceans, 2005.

[5] P. Newman, "MOOS: Mission Oriented Operating Suite", http://www.robots.ox.ac.uk/ pnewman/.

[6] Benjamin, M., J. Curcio, J. Leonard, and P. Newman . 2006. "ProtocolBased COLREGS Collision Avoidance Navigation Between Unmanned Marine Surface Craft" Journal of Field Robotics, Vol. 23, No. 5(May).

[7] Benjamin, M., J. Curcio, J. Leonard, and P. Newman. 2006. "Navigation of Unmanned Marine Vehicles in Accordance with the Rules of the Road" International Conference on Robotics and Automation (ICRA), Orlando FL, May 2006.

[8] Vaganay, J. J. Leonard, J. Curcio, and S. Willcox. 2004. "AOFNCexperimental validation of the moving long baseline navigation concept". AUV 2004.

[9] Delay Tolerant Networking Research Group (http://www.dtnrg.org)

[10] Nason, I; Puig-Suari, J; Twiggs, R. 2002. "Development Of A Family Of Picosatellite Deployers Based On The CubeSat Standard", In Proceedings of the 2002 IEEE Aerospace Conference Proceedings, Vol. 1, Big Sky, Montana, March 9-16 2002

[11] Heidt, H., J. Puig-Suari, A.S. Moore, S. Nakasuka, and R.J. Twiggs, 2001."CubeSat: A new Generation of Picosatellite for Education and Industry Low-Cost Space Experimentation" In Proceedings of the 14th Annual AIAA/USU Conference on Small Satellites, Logan, Utah, August 24-2, 2001

[12] Swartwout, M. 2004. University-Class Satellites: From Marginal Utility to 'Disruptive' Research Platforms. 18th Annual AIAA/USU Conference on Small Satellites. 11pp. 